\title{
Pertinencia y actualidad de la pedagogía amigoniana*
}

Pertinence and Topicality of Amigonian Pedagogy

Pertinência e atualidade da pedagogia amigoniana

\section{Luis Eduardo Corredor Vargas** (iD) https://orcid.org/0000-0003-1726-7759 \\ David Esteban Zuluaga Mesa*** (iD https://orcid.org/0000-0002-8975-5957}

\section{Doi: 10.17227/rce.num78-7045}

Para citar este artículo: Corredor, L. y Zuluaga, D. (2020). Pertinencia y actualidad de la pedagogía amigoniana. Revista Colombiana de Educación, 78, 229-252. https://doi.org/10.17227/rce. num78-7045.

\section{(ल) $(1) \Theta$}

* $\quad$ Este artículo forma parte del proyecto de investigación "Estudio nacional sobre el papel de la universidad y las instituciones amigonianas en la formación de los estudiantes universitarios y educadores sociales como agentes constructores de paz desde el enfoque del Desarrollo Humano en diez ciudades colombianas (Medellín, Apartadó, Manizales, Bogotá, Montería, Popayán, Pasto, Yopal, Tunja y Palmira)", ejecutado, durante 2017, por los grupos de investigación Educación, Infancia y Lengua Extranjera y Filosofía y Teología Crítica, de la Universidad Católica Luis Amigó y el Centro de Investigaciones Carisma y Saber de los Religiosos Terciarios Capuchinos Provincia San José.

** Magíster en Educación de la Universidad Católica Luis Amigó. Coordinador pedagógico de la Institución Educativa María Auxiliadora del municipio de Sabaneta. Correo electrónico: Iuiseduardo0828@gmail.com.

*** Doctor en Filosofía de la Universidad Pontificia Bolivariana. Editor de la revista Perseitas y líder del grupo de investigación Filosofía y Teología Crítica de la Universidad Católica Luis Amigó. Correo electrónico: david.zuluagame@amigo.edu.co. 


\section{Resumen}

Este artículo de revisión presenta un rastreo de la producción escrita en torno al desarrollo teórico de la pedagogía amigoniana. El proceso metodológico implicó, en un primer momento, la identificación de fuentes referentes al amigonianismo en general; así se obtuvieron 78 referencias (13 libros, 32 artículos, 22 actas de congresos, 8 boletines informativos, 2 tesis doctorales y 1 cartilla). Luego se hizo una revisión en la que se seleccionó únicamente el material que tuviera relación con la pedagogía amigoniana; como resultado se descartaron 22 fuentes y se conservaron 56. Estas se leyeron a profundidad y se identificó cómo se aborda en ellas puntualmente el campo pedagógico amigoniano en torno a su identidad, carisma, espiritualidad, principios, valores, actitudes y perfil del educador amigoniano. Se encontró que solo 40 de ellas desarrollan el tema que nos ocupa, por lo tanto se descartaron las 16 restantes. El ejercicio de revisión permitió identificar los pilares de la pedagogía amigoniana, los retos que debe atender en el mundo contemporáneo, sus principales discusiones y, sobre todo, indagar acerca de su pertinencia y su actualidad. El artículo concluye indicando que la finalidad de la pedagogía amigoniana es reflexionar, investigar, sistematizar y socializar su saber pedagógico, direccionando sus esfuerzos a la formación de agentes sociales, buscando transformaciones benéficas a través de su identidad y carisma como garante de los derechos fundamentales de las personas.

\section{Palabras clave}

educación correccional; justicia de menores; organizaciones religiosas; pedagogía; problemas de la juventud; programas de rehabilitación

\section{Keywords}

correctional education; juvenile justice; pedagogy;

rehabilitation programs; religious organizations; youth problems

\begin{abstract}
This paper reviews written production about the theoretical development of Amigonian pedagogy. First of all, the methodological process involved the identification of sources about Amigonianism in general, which resulted in 78 references (13 books, 32 papers, 22 conference proceedings, 8 newsletters, 2 PhD dissertations, and 1 passbook). Then, a review was made in which only material related to Amigonian pedagogy was selected; as a result, 22 sources were discarded and 56 were kept. An in-depth reading of these sources was then carried out, identifying how the field of Amigonian pedagogy is approached in terms of their identity, charisma, spirituality, principles, values, attitudes and profile of Amigonian educators. It was found that only 40 of them develop the topic at hand, and therefore the remaining 16 were discarded. The review allowed us to identify the pillars of Amigonian pedagogy, the challenges that must be addressed in the contemporary world, their main discussions and, above all, it allowed us to inquire about its relevance and its topicality. The paper concludes by indicating that the purpose of Amigonian pedagogy is to reflect on, research, systematize and socialize their pedagogical knowledge, guiding their efforts to the training of social agents, seeking beneficial transformations through their identity and charisma as guarantor of the fundamental rights of people.
\end{abstract}

\section{Resumo}

Este artigo apresenta uma revisão de produção escrita sobre o desenvolvimento teórico da pedagogia amigoniana. O processo metodológico implicou, em primeiro lugar, a identificação de fontes referentes ao amigonianismo em geral. Assim, obtivemos 78 referências (13 livros, 32 artigos, 22 atas de congressos, 8 boletins de informação, 2 teses doutorais e 1 cartilha). Depois realizamos uma revisão na que selecionamos unicamente o material relacionado com a pedagogia amigoniana. Como resultado, descartamos 22 fontes e conservamos 56. Estas foram lidas a profundidade e identificamos como nelas é abordado especificamente o campo pedagógico amigoniano em torno a sua identidade, carisma, espiritualidade, princípios, valores, atitudes e perfil do professor amigoniano. Encontramos que só 40 delas desenvolvem o tema questionado, portanto, descartamos as outras 16.0 exercício de revisão permitiu identificar os pilares da pedagogia amigoniana, os desafios que deve conquistar o mundo contemporâneo, suas principais discussões e, especialmente, indagar sobre sua pertinência e sua atualidade. $\mathrm{O}$ artigo conclui assinalando que a finalidade da pedagogia amigoniana é refletir, pesquisar, sistematizar e socializar seu conhecimento pedagógico, norteando seus esforços para sua identidade carisma como garante dos direitos fundementais das pessoas.

\section{Palavras-chave}

educação correcional; justiça de menores; organizações religiosas; pedagogia; problemas da juventude; programas de reabilitação 


\section{Introducción}

De acuerdo con Amigó (1986), González y Vives (1986), Vives (2000) y Abad Lara (2016), la raíz de la pedagogía amigoniana está en las experiencias de vida de su fundador, el padre fray Luis de Masamagrell, representadas en cuatro vivencias: 1) la formación católica cristiana que se vivía en su familia de cuna; 2) la reflexión de los evangelios a la luz del carisma de San Francisco de Asís cuando se vincula a la vida religiosa en el convento Perla de la Orden Capuchina, de Bayona, Francia, en 1874; 3 ) el bautizo de un niño recién nacido y abandonado en el convento de Montehano-España, en 1879; y 4) las prédicas y atenciones que ofrecía a los encarcelados como parte del carisma de los monjes capuchinos. Así,

La labor desarrollada en el penal de Santoña (Santander- España, a finales del siglo xIx), la frialdad y distancia que allí observó le impresionó vivamente $y$, con sus frecuentes visitas, su dulzura y paciencia, lograría convencer no sólo a su primer amigo Abilio, un montañés condenado a cadena perpetua, sino a algunos más. Y la paciente labor de todo un año, dio como resultado el que un buen número de reclusos cambiaran su manera de pensar y actuar. Esto llevó a Luis Amigó a pensar la forma de dar continuidad a la educación moral de los presos, con amor y caridad, ofreciéndoles un trato cercano, dedicándoles tiempo. (Abad, 2016, pp. 24-25).

Estas experiencias condujeron al padre Luis Amigó a la fundación de la Congregación de Religiosas Terciarias Capuchinas el 11 de mayo de 1885, "a las que indicó como principales campos de compromiso en su quehacer: la atención de enfermos, la enseñanza de niñas y jóvenes y, particularmente, el cuidado de los huérfanos" (Vives, 2000, p. 16), acción que fue motivada por las condiciones precarias que había dejado una epidemia de cólera que por esos días aquejaba a España ${ }^{1}$.

Posteriormente, el 12 de abril de 1889, el padre Amigó funda la Congregación de Religiosos Terciarios Capuchinos de Nuestra Señora de los Dolores y, "tras haberles indicado en un principio distintos campos de actuación social —les orientó, en 1890 — - [a] la labor de educar integralmente a los niños y jóvenes en conflicto" (Vives, 2000, p. 16),

1 "Pasada la epidemia de cólera, se vio que quedaban muchos niños sin amparo por haber muerto sus padres [...]. Y movido yo a compasión [...] pensé en que podíamos recogerlos, consulté el asunto a las juntas de la tercera orden y sin pérdida de tiempo alquilamos en Massamagrell, la casa llamada del castillo, para convertirla en asilo donde recoger a los niños huérfanos. Salimos luego por la población [...] a recoger algunos muebles que nos ofrecieron y con varias limosnas que me dieron compramos jergones, sábanas y sin contar con más recursos, pero confiados en la divina providencia, abrimos el asilo el 9 de agosto del mismo año 1885. Hacía tan solo una semana se había dado por finalizada oficialmente la epidemia de cólera en Valencia" (Amigó, 1986, pp. 45-46). 
intención que rindió frutos cuando en 1890 asumieron la dirección del Centro de Reforma Santa Rita España², en el que los Terciarios Capuchinos introdujeron importantes desarrollos y prácticas en los campos psicológico, pedagógico, psicopedagógico, legislativo (en función de los menores) y humanístico (orientado al humanismo cristiano).

Para los Terciarios Capuchinos, el Centro de Reforma Santa Rita es significativo por ser el primer centro reeducativo en el que se comienzan a desarrollar lo que se conoce hoy como pedagogía amigoniana, iniciando lo que denomina el padre Lostado (2015) como "un proyecto educativo [...] que abriría una manera nueva de hacerse presente en el mundo de la educación a través de los métodos y del estilo propio que ellos [los Terciaros Capuchinos] supieron implantar" (p. 12). Y que para esa época centraba su atención, según Mestre (2015), en "la enseñanza y moralización de los acogidos en las escuelas de reforma y correccionales" (p. 39).

En efecto, los Religiosos Terciarios Capuchinos fueron los protagonistas de lo que se nombra como la primera tradición pedagógica amigoniana ${ }^{3}$, Ilevaron y adaptaron al escenario educativo de la Escuela Santa Rita las directrices de su comunidad fundadas en el humanismo cristiano de los franciscanos, lo que configuraría la base primordial de esta especial y particular pedagogía y que posteriormente el padre Vives (2000) denominaría "identidad amigoniana", expresión que congrega la espiritualidad y el carisma amigoniano.

Teniendo esto como base, el propósito de este artículo es revisar en la literatura acerca de la pedagogía amigoniana cómo esta ha logrado ser una respuesta de atención, intervención y acto educativo para la población

2 "El primer centro de menores español denominado de tal modo fue el Asilo de corección paternal de Santa Rita [...]. Fue un centro de referencia a nivel nacional que se fundó por el senador Francisco Lastres al amparo de la Ley de 4 de enero de 1883, promulgada especialmente para Santa Rita" (Mestre et al., 2012, p. 58). La misión de esta institución era la educación de la juventud, por medio de la corrección paternal. Ingresaban varones mayores de 9 años a quienes los tribunales declaraban irresponsables y necesitaban medidas de tutela. Como señala Montero (2015, p. 69), esta escuela de reforma nace en 1875 como proyecto, cuando el Sr. Lastres se compromete ante un grupo de periodistas a crear un establecimiento correccional de jóvenes para atender las frecuentes reclamaciones de padres y tutores que no podían contener a sus hijos, y recoger a los menores más o menos abandonados, material o moralmente, por sus familias para evitar su ingreso en el mundo de la delincuencia.

3 "Se considera primera tradición puesto que es en esta época donde se cimientan las bases metodológicas de este postulado teórico, se le atribuye en gran medida la sistematización de esta experiencia al padre Domingo de Alboraya" (Martínez, 2009, pp. 5-8). De acuerdo con Vélez de la Calle (2009), "la sistematización de la experiencia psicopedagógica y la influencia de múltiples discursos disciplinares, consolidó la pedagogía reeducativa como la segunda tradición de la pedagogía amigoniana" (p. 4), "para ubicarnos en una actualidad no solo en lo reeducativo, también en lo preventivo, familiar, educativo, comunitario, jurídico, Parroquial y social, liderados por 400 Religiosos Terciarios Capuchinos y más de tres mil laicos amigoninos en 22 países de cuatro continentes" (Martínez, 2015, p. 31). 
infanto-juvenil que se encuentra en riesgo por la vulneración de derechos o por estar en conflicto con la ley penal. Esto se logra analizando textos clave, propios de la literatura de escritores amigonianos, que han permitido llevar el quehacer a la reflexión escrita, rescatando y configurando formas particulares de ver las raíces fundacionales y la actualidad misional del abordaje educativo-reeducativo, que ha presentado y reconfigurado constantemente una propuesta pedagógica acorde con las necesidades de la población.

\section{Metodología}

El rastreo de información acerca del conocimiento generado en torno al desarrollo teórico de la pedagogía amigoniana se hizo entre los meses de agosto de 2016 y agosto de 2017, fundamentalmente en bases de datos como: Fuente Académica y Fuente Académica Plus de Ebsco, Google Académico, Dialnet, Scielo y libros de memorias de congresos de pedagogía amigoniana en las que se consignan sus últimas posturas. El rastreo se hizo atendiendo a las siguientes categorías: pedagogía amigoniana, identidad, carisma y espiritualidad amigoniana.

El material consultado provino de 78 fuentes, distribuidas según su tipología: 13 libros, de los cuales 6 se configuraron como fuentes primarias y 7 como fuentes secundarias. 32 artículos, 22 actas de congresos, 1 cartilla, 8 boletines informativos amigonianos y 2 tesis doctorales. En ellos, los temas más recurrentes fueron pedagogía, identidad, carisma, espiritualidad, principios amigonianos, valores, actitudes, perfil del educador amigoniano, presencias del quehacer amigoniano en el mundo, entre otros.

La revisión inicial se hizo considerando, en primer lugar, título, resumen y palabras clave. Los textos que no tenían relación con la pedagogía amigoniana fueron descartados, los que sí pasaron a una segunda fase de lectura. El total de fuentes descartadas en este primer proceso de revisión fue de 22 (todos artículos), mientras que las 56 fuentes restantes pasaron a la segunda fase de lectura. Esta segunda fase consistió en hacer una revisión detallada del material con el fin de identificar la forma como en cada una de ellas se aborda el campo pedagógico amigoniano, en esta fase se descartaron 16 fuentes por lo que, finalmente, el desarrollo del artículo se hizo considerando las 40 fuentes seleccionadas.

Atendiendo a la selección final de textos, el estudio se centró en identificar autores, tesis o puntos de vista, conclusiones y referencias base, que se destacaron entre todo el material consultado como fuentes de primer orden (véase el anexo 1). 


\section{Contexto histórico y objeto de la pedagogía amigoniana}

La primera visita a la cárcel de Santoña (España) por parte del padre fray Luis Amigó sería la base para dar respuesta a la necesidad de atender población en condición de vulnerabilidad y conflicto con la ley. Para aquel momento la poca participación de los presos en la misa, sumado a que se consideraban peligrosos (el sacerdote tenía que celebrar la misa dentro de una cabina y estar altamente vigilado por guardianes), generó interés en el padre Amigó, pues, "mucho sintió el celoso misionero la frialdad que había advertido en aquella cárcel" (González y Vives, 1986, p. 28). Situaciones de las que se ocupó la naciente comunidad de Terciarios Capuchinos comisionada de atender tales dificultades del sistema carcelario.

Este fue el inicio de una forma de atención, acompañamiento y educación en la moral, dirigida a población carcelaria, que se conoció en un primer momento como ciencia del corazón humano, "por cuanto atendida la índole del corazón humano, el medio más hermoso para estimular a los niños, [hacia la] práctica de la virtud como a la aplicación al estudio o al trabajo" (Amigó, 1986, p. 642), práctica que mantuvo la atención no solo en los desarrollos psicopedagógicos, sino también en la sensibilidad para aprender, por la experiencia, la ciencia del corazón, como enfatiza Vives (2001, p. 29) y que redundaría más tardes en lo que se conoce hoy como pedagogía amigoniana.

La pedagogía amigoniana, según la conciben González y Vives, es la "ciencia y arte de la reeducación [...]. La reeducación en consecuencia, es la acción de educar de nuevo; la acción educativa se centra en el individuo caído, lesionado, extraviado, proporcionándole impulsos para que pueda realizar su vocación" (1986, p. 13). Esta propuesta pedagógica en reeducación ofrece una formación integral con sentido humano ${ }^{4}$ que, como enuncia Roa (2014):

4 La pedagogía amigoniana transitó en las bases conceptuales la pedagogía correccional durante gran parte del siglo xx, pedagogía orientada para los "anormales", concepción desarrollada más a profundidad por (Moreu, 2006; Venceslao, 2012). También vivenció desarrollos de la psicología experimental y psicopedagogía (Sánchez, Guijarro y Sanz, 2005; González, 2011; Mestre et al., 2012), donde los Terciarios Capuchinos diseñaron instrumentos experimentales al campo científico como la ficha biopsicopedagógica (González, 2011), psicopedagogía correccional (Vélez et al., 2008, p. 48) y fueron los mismos Terciarios Capuchinos quienes desarrollaron el constructo disciplinar de la pedagogía reeducativa en Colombia a finales del siglo xx (Vélez et al., 2008, pp. 24 y 89), para hoy configurarse y nombrarse como pedagogía amigoniana con significativa presencia en América (Religiosos Terciarios Capuchinos, 2012; Velasco, 2016), Europa, Asia y África (Del Peral, 2015, pp. 127-181). 
Va más allá de una instrucción o una educación formal académica, es dar y tener responsabilidad en cada acontecimiento social, es darle al ser humano un motivo y una razón de vivir en el mundo en el que se nació. (p. 21).

Lo anterior valió para que autores como León, Calderón y Romero (2010) ubicaran la pedagogía amigoniana en el ámbito de la educación social y la consideraran

... una propuesta pionera de la pedagogía social, porque emergió en una época en la que si bien es cierto existía ya una preocupación en los países más desarrollados por atender a los niños y adolescentes en conflicto con la ley, aún estaban en ciernes las bases de una pedagogía y/o Educación que abordara en forma específica y concreta a los sujetos que requerían un tratamiento especial por estar en situación de discordia social. (p. 62).

Esto se da, porque la pedagogía amigoniana, a diferencia de los enfoques pedagógicos basados en la psicología, la sociología, la biología, entre otras orientaciones empleadas para apoyar el acto educativo, apela al saber filosófico y teológico a través del humanismo cristiano de los franciscanos capuchinos $^{5}$, que acogen el sentido de servicio social por el más necesitado, especialmente a los jóvenes y marginados como se evidencia en el Manual pedagógico de los Terciarios Capuchinos (1985), y en las obras de Amigó (1986), Vives (2001) y León et al. (2010). Es por ello que durante la revisión teórica a propósito de la pedagogía amigoniana se encuentra, con frecuencia, la utilización de pasajes bíblicos como estrategias para atender cuestiones propias del ser y el quehacer de la pedagogía amigoniana. De este modo, el Nuevo Testamento, las parábolas del evangelio cristiano, las bienaventuranzas, las expresiones de Jesús y los pasajes de la virgen María acompañando a Jesús cobran protagonismo y son usados en la literatura amigoniana para explicar el fondo antropológico cristiano, filosófico, existencial y práctico de esta pedagogía.

La antropología a la que hace referencia esta pedagogía es propia de la cultura cristiana, según la cual "el hombre fue creado a imagen y semejanza de un Dios, cuya verdadera identidad es el amor", como lo sugiere Vives (2001). Una antropología que reconoce al hombre, según León et al., como

5 La comunidad religiosa de los Franciscanos se dividen en tres agrupaciones: franciscanos conventuales, caracterizados por la contemplación de las cosas celestiales; franciscanos observantes, cuya identidad procura el ser misionero o activos al servicio del prójimo, y los franciscanos capuchinos con identidad mixta, o sea que tiene como fin lo celestial (Dios) y al prójimo (sociedad) (Amigó, 1986, p. 791), de estos últimos es donde se gesta la pedagogía amigoniana: Religiosas y Religiosos Terciarios Capuchinos. 
... un ser integral, sujeto protagonista de su propia vida, miembro vivo de un contexto histórico ambiental, con potencialidades y limitaciones [...]. Una persona con capacidad para enfrentar las adversidades de la vida, superándolas y saliendo de ellas fortalecido o con la fuerza necesaria para trasformar su propia realidad. (2010, p. 7).

Es a partir de esta mirada particular de ser humano integral que se establece el puente entre sujeto e intención pedagógica, o sea, hacia quién y para dónde se dirige el acto educativo. Así,

... el sistema pedagógico amigoniano trabaja integralmente todas las dimensiones de la persona, es decir, que la entiende como un ser biopsicosocial, que con su inteligencia incorpora y desarrolla actitudes que desde la ética y la moral conducen su dimensión racional. (León, Calderón y Romero, 2010, p. 8).

La población a la que ha apuntado la pedagogía amigoniana, como objeto y sujeto de la atención e intervención, ha sido la niñez, de preferencia marginada, específicamente aquellos niños que se encuentran en condiciones de vulnerabilidad o en conflicto con la ley penal. En ellos hay unas características comportamentales y existenciales particulares, que el Obispo Luis Amigó (1986) enmarco en la idea de "juventud apartada de la verdad y el Camino" ${ }^{\prime 6}$. Estas personas

Han experimentado privaciones o carencias materiales, afectivas, culturales, espirituales; [...] han recibido pobres formas de identidad, modelos negativos de imitación, influencias corruptoras [...] adolescentes que han crecido cronológicamente pero no psicológica ni espiritualmente [...] y presentan rasgos característicos de inseguridad, inestabilidad, violencia, ignorancia, agresividad. (Terciarios Capuchinos, 1985, p. 23).

Así las cosas, la "marginalidad" no se asume con una connotación despectiva o peyorativa, por el contrario, alude a la mirada franciscana de hacer presencia humana por medio del acto educativo y formativo atendiendo a "esa ternura personalizada que impulsa a amar allí donde hay mayor carencia o necesidad" (San Francisco de Asís, citado por Vives,

6 Entendido también como "niño inadaptado", "joven marginado" o "muchacho infractor" (Terciarios Capuchinos, 1985, p. 23). La delincuencia juvenil como concepto debe ser entendida desde diferentes miradas: la jurista, que contempla a ese ser humano que infringe una ley penal; la psicológica, que obedece a una serie de factores preponderantes y otros desencadenantes; la sociológica, que explica el fenómeno desde condiciones sociales; la pedagógica, que ve al delincuente como el resultado de una serie de circunstancias que ha vivido el sujeto (enfermo de conducta con derecho a tratamiento), y la del educador amigoniano que quita toda categoría jurídica para "acoger y proporcionarle las atenciones que necesita, a fin de que pueda integrarse plenamente a su mundo social" (Terciarios Capuchinos, 1985, p. 30). 
2001, p. 36), es por eso que la pedagogía amigoniana cree y busca rescatar, desarrollar y potencializar el ser humano destinado a trascender, con independencia de sus situaciones o dificultades particulares.

Pensadores como Mestre (2015) y Sesé (2015) comparten el pensamiento del padre Vives (2001), quien expresa que este movimiento social amigoniano es relevante por la labor de educar integralmente a los niños y jóvenes en conflicto, asunto que todavía hoy tiene relevancia y distintos escenarios de aplicación, como indican León et al. (2010), quienes exponen los espacios donde se desarrolla la propuesta amigoniana, a saber:

» Programas de prevención: Dirigidos a niños, adolescentes, jóvenes y sus familias, con el fin de "reconocer, analizar y resignificar factores generativos y de riesgo [...] y de prevenir eventos que deterioren la calidad de vida e impidan un proceso de socialización armónico" (p. 72).

» Programas de protección: Destinado a la niñez y los adolescentes "que se encuentran en grave peligro físico y moral por situaciones de abandono y desprotección familiar [...] a fin de favorecer un adecuado desarrollo bio-psicosocial, afectivo y moral" (p. 72).

» Programas de reeducación: Ligados al sistema jurídico y penal, atienden las medidas legales asignadas por las autoridades y entes territoriales responsables de la administración de justicia a chicos en conflicto con la ley, enmarcados en los designios legislativos internacionales y nacionales, "implementando con los equipos interdisciplinarios de profesionales en varias de las ciencias sociales y humanas, procesos integrales [...] que favorezcan la inserción efectiva de los niños, niñas, adolescentes o jóvenes en su medio familiar y social" (p. 72).

»Programas de comunidades terapéuticas: Brindan tratamiento pedagógico para afrontar y enfrentar la problemática del consumo de sustancias psicoactivas o las conductas que causan dependencia.

» Programas de formación para el trabajo: Se orienta en áreas de interés y se aporta formación para la vida, "valores, desde la actividad física, cultural, deportiva y el conocimiento de los deberes y derechos del trabajador" (p. 73).

»Colegios amigonianos: En los que se brinda lo concerniente a la educación formal.

» Parroquias: Promueven la armonización de las relaciones sociales y el fortalecimiento del tejido social.

Esto permite entender que las realidades sociales son dinámicas, mutables y cada vez más complejas, pues emergen aceleradamente nuevas problemáticas, producto de las inequidades, las injusticias, la disfuncionalidad 
familiar, la falta de conciencia de las personas, la ineficacia del Estado y de los sistemas políticos asociados a él, ausencia y falta de herramientas en el sistema educativo tradicional que atiendan e intervengan retos sociales como el uso y abuso de sustancias psicoactivas, la violencia intrafamiliar, la delincuencia juvenil y organizada, la deserción escolar y, en general, los comportamientos y actitudes que trasgreden y vulneran los derechos fundamentales de los seres humanos. Es en estas situaciones donde la pedagogía amigoniana logra tener asidero en cuanto respuesta y alternativa que se actualiza constantemente, de allí que no sea nombrada como un modelo pedagógico, sino como sistema pedagógico que posibilita y continúa afrontando el reto de educar y reeducar a la población marginada, excluida, problematizada, pues es un saber pedagógico que se fundamenta en la dignificación del ser humano y que permite a los sujetos dejar de justificar sus comportamientos y estilos de vida al margen de la legalidad, en las condiciones de vida particulares que deben afrontar ${ }^{7}$. El educador de esta pedagogía, como indica González (2004), es "aquel profesional que interdisciplinariamente brinda su ser y saber en la atención e intervención de los menores de edad" (p. 146), está preparado para atender al ser humano funcional como a ese otro ser humano conflictivo o en conflicto, en función de que resignifiquen su proyecto de vida disminuyendo los riesgos de vulnerabilidad.

\section{El papel de la pedagogía amigoniana ante la vulnerabilidad y contexto normativo en Colombia}

La vulnerabilidad es la condición constante de riesgo a la que está sujeto el ser humano y la carencia de las condiciones para afrontar, superar o reducir esos riesgos (Feito, 2007). Frente a esta consideración, la pedagogía amigoniana tiene como principal objetivo no solo ofrecer herramientas para afrontar, superar o reducir las condiciones de vulnerabilidad, sino también, velar por el restablecimiento de los derechos de la población vulnerable. En razón de ello, la labor formativa de la pedagogía amigoniana debe articular los principios fundacionales de la comunidad de los Religiosos Terciarios Capuchinos con la normatividad nacional que permita garantizar el restablecimiento de derechos de las personas implicadas en el proceso formativo.

7 La población en conflicto con la ley tiende a justificar sus comportamientos en excusas como: disfuncionalidad familiar, padres abandonantes, condiciones económicas desfavorables, madres trabajadoras sexuales, maltrato, resentimiento, abuelas permisivas, falta de oportunidades, contextos violentos, profesores exigentes, entre otras. 
Para el caso de Colombia, esta articulación implica la consideración del sistema nacional de bienestar familiar en el que Durán, Guáqueta y Torres (2011) abordan el restablecimiento de derechos rescatando el lugar jurídico y pedagógico desde el marco de la convención internacional de los derechos del niño (1989), el artículo 44 de la Constitución Política de Colombia (1991), los primeros artículos del Código de Infancia y Adolescencia (Ley 1098 de 2006), así como los fines de la educación expresados en la Ley 115 de 1994 y la Ley 1878 de 2018, en las que se indica que un menor de edad está en condición de vulnerabilidad cuando se violan sus derechos fundamentales o cuando no se garantiza el cumplimiento de los mismos.

Atendiendo a lo anterior, el Sistema Nacional de Bienestar Familiar (SNBF) y el Instituto Colombiano de Bienestar Familiar (ICBF) emiten la Resolución 1519 del 23 de febrero del 2016 y las subsiguientes resoluciones que la modifican, procurando conservar la corresponsabilidad del Estado y la sociedad en el restablecimiento de los derechos de los menores de edad, bajo parámetros o lineamientos técnicos, a fin de superar la condición de vulnerabilidad.

En cuanto al Sistema de Responsabilidad Penal para Adolescentes (SRPA), el contexto es similar, pues siempre se busca el interés superior del menor de edad (art. 8, Ley 1098 de 2006), cuya única variación está representada en que el adolescente estará inscrito en un proceso de judicialización por la presunción o responsabilidad en la comisión de un delito, donde las acciones pedagógicas están guiadas al restablecimiento de derechos, pero dentro de los parámetros de acciones reparadoras y restaurativas ante su trasgresión a la ley, como se evidencia en los artículos 139 y 140 de la Ley 1098 de 2006.

Desde esta perspectiva, la pedagogía amigoniana ofrece una alternativa de atención e intervención interdisciplinaria en pro de la formación y el restablecimiento de derechos de este grupo poblacional y desde allí su profunda relación con las políticas del Estado colombiano y su campo de acción.

Por su parte, como se evidencia en la revisión de los textos consultados para el desarrollo de este artículo, la espiritualidad, los principios y los ejes filosóficos amigonianos se interrelacionan y fortalecen en cuanto parten del referente de Jesucristo como maestro (), la madre de los dolores como fuente de misericordia y fortaleza), y el carisma franciscano que invita a colaborar en la restauración del hombre y la donación sin recompensa (Martínez M., 2009, Vives, 2000), elementos que, para Abad (2016), redundan en la tarea del maestro y propenden por la aplicación de tres principios:

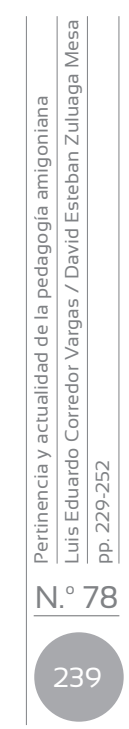


1. Esperar siempre la recuperación del joven, creer en la bondad natural de toda persona.

2. Educar con un sentido realista de la existencia, fortalecer su desarrollo personal para afrontar las dificultades.

3. Tratar a los muchachos con afecto y cariño, mostrando preocupación, compromiso y siempre dentro de un ambiente de acogida natural y afectuoso, aceptando y respetando su procedencia familiar y ambiental, con un tratamiento individualizado según sus necesidades (Abad, 2016, p. 39).

Lo que implica, como señala Sesé (2015), que el educador amigoniano debe "observar para conocer [y] conocer para educar" (p. 98); y esto, en articulación con los tres principios ofrecidos por Abad, invita, como señala Vives (2015), "a no perder nunca la esperanza en la feliz recuperación de cada uno de sus alumnos, e incluso a esperar, contra toda humana esperanza, en que no hay ningún caso imposible" (p. 92, cursivas del original ).

Así las cosas, el acto pedagógico que subyace de la pedagogía amigoniana está mediado por el amor y esto implica "amar a quien más lo necesita" (Arive, 2015, p. 51), que sintetiza "quien no ha sido querido, no puede querer, no se puede querer, no sabe quererse" (Arive, 2015, p. 49). De ahí que el amor sea esencia y la felicidad referente de la pedagogía amigoniana (Martínez C., 2015, p. 23), idea que, como refiere Osoro (2015), funde sus raíces en "la capacidad de amar y de entregar la felicidad a los demás" (p. 33): legado a los amigonianos, de parte de fray Luis Amigó, a través de su obra.

Por otro lado, Arive (2015) desarrolla la idea de la importancia que tienen las actitudes del educador amigoniano cuando se habla de intención reeducativa y educativa, pues, según él, "para la pedagogía amigoniana, para los amigonianos, los niños y adolescentes con problemas, desadaptados, son los que constituyen nuestra razón de ser y hacer, 'en ellos, por ellos y para ellos' adquiere valor y sentido cuanto somos y hacemos" (pp. 51-52). Es por ello que el educador amigoniano debe tener siempre presente como eje pedagógico lo siguiente:

» [El] valor supremo de la persona, de cada niño.

»Educar desde el sentimiento. Educar para la responsabilidad.

» [Educar] desde la cercanía, el amor.

» Ayudar al niño en su desarrollo completo formando la totalidad de sus potencialidades.

» Formar en libertad y desde la libertad, progresivamente y a la medida.

» Hacer al chico protagonista de sus conquistas y progresos integrados en sus procesos educativos en cada momento de la vida. 
» Creer en todo niño. No hay ningún caso perdido del todo.

» Todo niño tiene derecho a ser querido y respetado en su intimidad y en todos sus derechos. (Arive, 2015, p. 62).

Elementos que, para Osoro (2015), incitan a considerar, además de los libros, la vida. Invitan a habitar el mundo, a ser comunidad con el otro, asumiendo que

La fuente de la que vosotros bebéis es esa sabiduría que es lúcida, que es penetrante, que es bondadosa, que es benéfica, que es amiga del ser humano, que es firme, que es segura, que es serena, que es vigilante, que penetra el corazón y transforma el corazón y la vida y es una sabiduría que nace del amor mismo en nuestro Señor. (p. 34).

No obstante, a pesar de beber de la misma fuente, las instituciones amigonianas no evidencian unanimidad en los valores que caracterizan su pedagogía, en cuanto estos se han determinado con el transcurrir del tiempo y de acuerdo con el perfil de la acción reeducativa del contexto en el que se desenvuelven. Por ejemplo, los valores que identifican la Institución Educativa de Trabajo San José son honestidad, responsabilidad, respeto, tolerancia, solidaridad y sentido de pertenencia. Por su parte, los de la Universidad Católica Luis Amigó son ética, solidaridad, justicia social, participación, convivencia armónica y gestión. Elementos que están determinados sustancialmente por el tipo de servicios educativos que prestan y el público al que se dirigen.

Desde el punto de vista teórico, Vives (2015) congrega el sentido e importancia del acompañamiento en el acto educativo resaltando "la actuación de Cristo -[que en] su pedagogía- [lo] tuvo, como uno de los valores más característicos e identificantes" (p. 87), para lo cual, resalta seis valores que desarrollan un buen acompañamiento del educador amigoniano:

1) Empatía: Llegar a conocer [...] mediante la diaria convivencia, el cordial compartir y la franca relación personal entre educadores y alumnos [...]. 2) Cercanía: De la constante presencia, del compartir descomplicado, sencillo y alegre [...], haciendo posible así [...] un verdadero ambiente de familia en el cotidiano convivir [...]. 3) Personalización: Para individualizar la acción educativa, procurando ofertar a cada alumno un "tratamiento a la medida" [...], con sus potencialidades y carencias [...]. 4) Generosidad: Capaz de desvivirse cada día, a ejemplo del Buen Pastor, por aquellos que le han sido confiados para que tengan vida en abundancia [...]. 5) Coherencia y Testimonio: Para "convertirse [...] en camino para sus alumnos, al hacerse, para ellos, personas creíbles por su integridad [...]. 6) Fortaleza: También denominado resiliencia, imprescindible, para no huir ante las dificultades [...] que, día a día, trae consigo todo proceso educativo. (Vives, 2015, pp. 87-90). 
Arive (2015), por su parte, no habla de valores, como lo hace Vives (2015), si no de las actitudes que debe desarrollar todo educador amigoniano a la hora de interactuar con los integrantes de la comunidad educativa a fin de que esta pedagogía se convierta en práctica permanente entre los servidores amigonianos. Define seis actitudes:

1) Apertura: Significa 'ser patente', estar ahí con toda su humánitas, con toda la grandeza, dignidad e indigencia propia de la existencia humana. 2) Acogida: Consiste en el recibimiento con cariño, simpatía, amabilidad, alegría y atención a todo cuanto necesite el estudiante, una vez haya Ilegado a una institución amigoniana. 3) Fortaleza: Refleja la esperanza y capacidad de amar del educador. 4) Comunicación-escucha: Favorece las buenas relaciones y la aceptación, la ayuda, pues se sabe escuchado, comprendido y tenido en cuenta. 5) Convivencia: Consiste en compartir con los estudiantes, de palabra y obra, sus ansias, luchas, deseos, penas, ilusiones, esperanzas, proyectos, recuerdos y experiencias. 6) Empatía: Es esa capacidad de ponerse en el lugar del otro. Comprender, sentir qué piensa, cómo se siente (Arive, 2015, pp. 58-59).

Sin embargo, más allá de los valores que subraya Vives y de las actitudes que expone Arive, la discusión actual sobre el tema que nos ocupa precisa que se planteen dos preguntas fundamentales para el quehacer de los maestros: 1. ¿Cómo estamos estableciendo nuestras relaciones interpersonales?, y 2. ¿Cómo las estamos Ilevando a los escenarios educativos? Loizaga (2015) realiza desarrollos teóricos en torno a los apegos seguros y los dependientes que se dan a partir de las relaciones o vínculos establecidos por cada ser humano; invita a la comunidad amigoniana a pensar y generar siete pilares que sustenten el apego seguro en los entornos educativos y que coadyuvan al desenvolvimiento "adecuado" de las relaciones interpersonales, están en articulación con los tres principios de Abad (2016), los seis valores de Vives (2015), las seis actitudes expuestas por Arive (2015) que propenden por los ocho ejes pedagógicos propuestos por el mismo Arive (2015) y que redundan en una productiva relación en escenarios educativos.

Primero, la capacidad de querer a los otros. "Esta capacidad incluye vínculos como dar consuelo, apoyo, confianza, cariño o cuidado positivo" (Loizaga, 2015, p. 120). Está en sintonía con la importancia de uno de los fundamentos madre de la pedagogía amigoniana "amar a quien más lo necesita" (Arive, 2015; Martínez C., 2015; Mestre, 2015; Osoro, 2015); con los valores 1, 2, 3 y 4 de Vives (2015, pp. 87-88); con las actitudes 1, 2, 3 y 4 (Arive, 2015, p. 58), y con los ejes pedagógicos 1, 2, 3, 7 y 8 (Arive, 2015, p. 62).

Segundo, la fuerza de los límites. "Los límites incluyen orientar o contener posibles actitudes y comportamientos que pueden ser dañinos para uno mismo o para los otros" (Loizaga, 2015, p. 120), esta fuerza 
comulga con el principio amigoniano 2 (Abad, 2016, p. 39), y con las actitudes 4 y 5, junto con los ejes pedagógicos 2, 4, 5, 6 y 8, propuestos por Arive (2015, p. 62).

Tercero, la fuerza del respeto. "Consiste en entender la diferencia y su diversidad y en la creencia de que todo ser humano posee un potencial importante para crecer y desarrollarse" (Loizaga, 2015, p. 120). Esta clave es desarrollada con intensidad en los postulados amigonianos en el valor 1, 3 y 7 (Vives, 2015, pp. 87-90) en la actitud 1, 2, 4, 5 y 6 (Arive, 2015, pp. 58-59) que cobran sentido en el eje pedagógico 1, 2, 3, 5, 6, 7 y 8 (Arive, 2015, p. 62).

Cuarto, la fuerza del modelo. "Toda persona puede ser modelo [...], especialmente cuando nos proporcionan ánimo para actuar, ayuda presencial o ejemplo directo o indirecto" (Loizaga, 2015, p. 120). En la tradición amigoniana, es en definitiva el sentido que se tiene de educador independientemente de la profesión, en cuanto a su relación con el valor 1, 2, 5 y 6 (Vives, 2015, pp. 87-90); con las actitudes 1, 2, 3, 5 y 6, y los ejes pedagógicos 2, 5, 6 y 7, propuestos por Arive (2015, pp. 58-62).

Quinto, la fuerza del lenguaje. "La comunicación hablada, la reflexión y la discusión respetuosa generan criterios y guías de actuación ante la vida. Por eso, la conversación educativa, la reflexión con nuestros educadores y la crítica positiva ayuda a entender a los otros" (Loizaga, 2015, p. 120). Esta clave encuentra relación con el principio amigoniano 3 (Abad, 2016, p. 39); los valores 1, 2, 3 y 5 (Vives, 2015, pp. 87-89); las actitudes 2, 4, 5, 6 , en coherencia con los ejes pedagógicos 2, 3, 4, 5 y 6, propuestos por Arive (2015, pp. 58-62).

Sexta, la fuerza del perdón y la fuerza del agradecimiento. "Ser capaces de entender quién nos ha producido daño, entender los motivos de sus actuaciones y perdonarle entendiendo sus fortalezas y debilidades. Pero, además, ser capaz de agradecer por aquello que acertaron y sobre todo reconocer el buen trato" (Loizaga, 2015, p. 121), clave que se relaciona con los valores 1, 2, 3 y 4, con fuerte acento en el sexto valor, formulado por Vives (2015, pp. 87-90), y las actitudes 1, 3, 4, 5 y 6, en coherencia con todos los ejes pedagógicos propuestos por Arive (2015, p. 62).

Estos principios, valores, actitudes del quehacer de la pedagogía amigoniana, permiten delimitar la relación profunda e importante del educador y sus alumnos, del alumno con sus pares, y en general de las relaciones interpersonales en las comunidades educativas amigonianas, de lo cual el padre Domingo de Alboraya, citado por Martínez M. (2009 ) y Abad (2016), fue uno de los gestores, pues lo que en un primer momento fue denominado ambientes reeducativos, continúa vigente hoy en los sistemas 
educativos amigonianos, solo que ahora aparece en ámbitos plurales, reflexionado y actualizado conforme a los tiempos. Ejemplo de ello es la actualidad de la pedagogía amigoniana en Europa.

Es importante educar en

... controlar las emociones, la conducta, la impulsividad; brindarles libertad y autonomía, enmarcadas siempre dentro del cariño y las exigencias $[\ldots]$ hábitos que, como indica Loizaga, se van creando hasta que se integran automáticamente en el cerebro del niño o joven. (Marina, 2015, p. 123).

Regina del Peral (2015) expone que la pedagogía amigoniana "implica vivir el sentido del servicio y el sacrificio, al punto de dar la vida por otros". Esto, según Anno (2015), implica vivir con la gente en el barrio: "Intervención desde la humildad y sencillez; entrar en relación, ganándose la confianza de los muchachos a base de simpatía y estimación; entrar en contacto con las familias y vecinos, y trabajar en red y fomentar el voluntariado" (p. 128).

Asuntos que, según Hoffend (2015), favorecen el trabajo con el público que llega a las instituciones amigonianas que, por lo general, manifiesta dificultades en los ámbitos judicial, psiquiátrico y educativo. Esto permite la programación de actividades en beneficio del proceso de los actores principales representadas en clases, estudio dirigido, trabajo físico en casa (sobre todo en limpieza y cuidado de las instalaciones), charlas individuales y grupales, sesiones de socioterapia, que exigen un trabajo compartido, un trabajo en red que, como plantea Tobón (2015), aporte a la innovación socioeducativa en la que se asuma la identidad amigoniana y pedagogía amigoniana como un método, un estilo de relación educativa trasformadora.

Por otro lado, atendiendo a las experiencias actuales de la pedagogía amigoniana en América, Rodríguez insiste, desde su experiencia en Argentina, en "la capacidad de la pedagogía amigoniana como herramienta para formar personas autónomas con éxito" (2015, p. 129). En Puerto Rico, se atiende en el Centro Politécnico Amigo estudios acelerados para "jóvenes de 16 a 21 años con ingresos bajo el umbral de pobreza" (Torres y Vélez, 2015, p. 129), y que presentan deserción escolar o en riesgo de serlo. En Querétaro (México), se desarrolla un proyecto de atención para niños y adolescentes en situación de riesgo, vulnerabilidad y a sus familias, con una propuesta pedagógica amigoniana integral (Pérez, 2015). Juan José Calderón (2015) expone la importancia de hacer las adaptaciones que ha hecho la pedagogía amigoniana para poder atender a los niños, niñas y adolescentes en condiciones vulnerables, expósitos con mayores dificultades en la provincia San José (Brasil, Colombia y Ecuador). Calderón expuso los proyectos realizados en la Provincia de San José, haciendo 
referencia a los "retos asumidos (las transformaciones del tejido social, una pedagogía crítica para la emancipación del sujeto en la sociedad de consumo y el protagonismo amigoniano en el surgimiento del Estado de Derecho)" (Calderón, 2015, p. 130). Este mismo autor narra que solo en Colombia hay 45 instituciones entre las que se encuentran de educación, reeducación, protección, prevención, comunidades terapéuticas y de educación superior.

Finalizando este tránsito de presencias de la pedagogía amigoniana en el mundo, cabe nombrar la experiencia de Friendship Home Father Luis Amigó, institución amigoniana presente por más de 25 años en Manila-Filipinas (Asia), donde ha logrado un reconocimiento local por una educación: "basada en el clima de familia, el acompañamiento personalizado y la potenciación de los proyectos personales [...] que no ha pensado en el impacto meramente individual del menor, sino que su extensión ha llegado también a su familia y comunidad" (Ortiz y Fagua, 2015, p. 177).

\section{Conclusiones}

"La pedagogía busca comprender las finalidades de la educación" (Zambrano, 2015, p. 45), que, en el caso de la pedagogía amigoniana, están en la intención de promover y ser garante de los derechos fundamentales de la niñez desde sus propuestas preventivas, educativas y reeducativas, abordajes que se complejizan entre el debate de la naturaleza y libertad del que habla Zambrano: porque "la naturaleza y la libertad tropiezan cuando educar entra en el registro de la cultura y de lo social pues supone el aprendizaje del conjunto de valores, normas, saberes legítimos y necesarios para su devenir" (Zambrano, 2015, p. 50).

Educar es una tarea que compete, en primera instancia, a la familia y, en segunda instancia, a la institución: "escuela". No obstante, y en esto asentimos con el expresidente de Uruguay José Mujica, no se puede dejar la responsabilidad formativa solo a la escuela, "no le pidamos al docente que arreglen los agujeros que hay en el hogar" (Mujica, 2014, párr. 1). Los agujeros o vacíos educativos hacen parte de las realidades sociales son inherentes al ser humano, son punto de partida para el surgimiento de las ciencias de la educación, de la pedagogía social y de otros postulados pedagógicos, es por ello que las instituciones deben atenderlos con la responsabilidad de formar o educar, tanto en saberes pedagógicos como cívicos, se debe procurar la formación de sujetos políticos.

La pedagogía amigoniana responde a los dos atributos epistemológicos fundamentales para considerarse saber pedagógico en la medida en que delimita el "quién", o sea, el sujeto de la intención educativa (menores 
de edad), en condición de vulnerabilidad y define el "qué" (Zambrano, 2015, p. 49), con el que se precisa el saber disciplinar específico (pedagógico), dando paso al saber didáctico, pues "la didáctica fija sus límites en el aprendizaje" (Zambrano, 2015, p. 45), propiamente en el "cómo": la didáctica.

El sistema pedagógico propuesto por la pedagogía amigoniana es "el currículo", "guía principal [que] se construye en el hacer educativo" (Álvarez y González, 2002, p. 78). El currículo es "la manera práctica de aplicar una teoría pedagógica al aula, a la enseñanza real" (Flórez, 2005, p. 291), y también define que "el modelo pedagógico social [es el] inspirador de un currículo que proporciona contenidos y valores para que los alumnos se formen en medio de la sociedad y ayuden a la reconstrucción social de la misma" (p. 295). Aquí se encuentra una base fundamental para hacer comprensible que hoy es importante y vigente la pedagogía amigoniana, tanto por su saber construido históricamente como por su misión, sus presencias (carisma y escenarios) y sistema aplicados al quehacer de la formación integral.

Este sistema pedagógico amigoniano formula "un método progresivo y secuencial por periodos [...] sustentado en un sistema de auto corrección y de acompañamiento" (León, Calderón y Romero, 2010). Están planteados en tres etapas del proceso pedagógico: "Acogida, tratamiento y postinstitucional ${ }^{8 "}$, aclarando que dentro de la etapa de "tratamiento" hay tres niveles de crecimiento personal: "encauzamiento, afianzamiento y robustecimiento" (p. 62).

Esta estructura del sistema pedagógico amigoniano favorece la generación de entornos protectores en tanto se logren climas y ambientes reales de familia, donde se puedan gestar ambientes de resignificación y reconfiguración de los proyectos de vida. De esta manera, la institución "escuela" deja de ser un entorno ajeno, distante e informativo, para ser continuador de la tarea de educar para la vida (sentirse en casa) y por ende formar se convierte en un proceso del ser durante la vida, para la vida y del que participan diferentes actores:

En la casa se aprende a: saludar, dar las gracias, ser limpio, ser honesto, ser puntual, ser correcto, hablar bien, no decir groserías, respetar a los semejantes y a los no tan semejantes, ser solidario, comer con

8 Etapa de Acogida: espacio de bienvenida y motivación que permite la inserción del niño, niña, adolescente y su familia dentro de un proceso reeducativo y/o protectivo-preventivo a través del medio institucional. Etapa de tratamiento: gestión de atención y formación integral (diagnóstico integral, elaboración y ejecución del plan de acción reeducativo, preventivo y/o protectivo a nivel individual, familiar y de preparación para el egreso). Etapa de postinstitucional: instancia de seguimiento y acompañamiento que permite evaluar y medir el impacto del proceso pedagógico- terapéutico (León, Calderón y Romero, 2010, pp. 65-66). 
la boca cerrada, no robar, no mentir, cuidar la propiedad y la propiedad ajena, ser organizado. En la escuela se aprende: matemáticas, lenguaje, ciencias, estudios sociales, inglés, geometría y se refuerzan los valores que los padres y madres han inculcado en sus hijos. Muy difícil es, hacer que el latón brille como el oro. (Mujica citado por Berberián, 2015, párr. 1).

Frente a este panorama, la pedagogía amigoniana asume el reto y la tarea de volver a educar en asuntos que, en primera instancia, eran competencia de la familia y el Estado — desde la garantía de derechos- La reeducación es, para la pedagogía amigoniana, su principal apuesta. Es a partir de esta que se revitaliza y actualiza, pues las sociedades latinoamericanas heredaron de la justicia retributiva la creencia del castigo disfrazado de sanción, como elemento de control social o escarmiento para que otras personas no repliquen dichos comportamientos. Lo anterior ha dado como resultado hacinamiento carcelario, corrupción, delincuencia organizada, violación a los derechos de los seres humanos, perpetuación de conductas punibles, disfuncionalidad social, inequidad, injusticia e impunidad. Estos resultados deben hacernos pensar acerca de la forma en que se educa y se castiga con relación en cómo se educa y cómo se corrige.

Es sabido que la educación es el camino más efectivo para trasformar el tejido social, que hasta la actualidad sopesa y fomenta sujetos aportantes a una sociedad. Es a partir de ella que podemos aventurarnos a pensar en las sociedades que tendremos "porque allí se anticipa el rostro de la sociedad que vendrá. [Pues] de la educación dependen buena parte de las potencialidades productivas de un país. [Como] también la futura aptitud de nuestra gente para la convivencia cotidiana" (Mujica, 2010, p. 8).

La pedagogía amigoniana es pionera, aportante y promotora en los escenarios sociales internacionales, pues la problemática delincuencial juvenil es una realidad social presente en el mundo, ocasionada por diversas causas y generadora de múltiples problemáticas que lesionan las comunidades. Estos problemas mutan en situaciones complejas de inseguridad social, violencia en todas sus formas y condiciones de miseria social, lo que condena a las sociedades actuales y futuras a trasegar arrastrando con sujetos enfermos en su conducta y sus vínculos sociales. Por ende, las repuestas no están en los actos jurídicos represores, sino en las acciones preventivas, educativas y reeducativas que propician inclusión social, la resignificación y formulación de proyectos de vida funcionales, que permitan el reintegro de las personas a la sociedad, la no reincidencia, como sujetos garantes de derechos y deberes.

En la actualidad, así como en sus orígenes, la pedagogía amigoniana requiere ser pensada de cara a contextos concretos como una alternativa de atención a la juventud vulnerable, máxime hoy que la comunidad 
académica tiene el reto de pensar en el posconflicto y esto exige escenarios alternativos en los que la rigidez de los manuales de convivencia brinden oportunidades de aprendizaje, y requiere herramientas pedagógicas capaces de trasformación social para las relaciones incluyentes.

El reto que asume hoy la pedagogía amigoniana está en reflexionar, investigar, sistematizar y socializar su saber pedagógico, en pro de acercarla a escenarios de interlocución, como instituciones educativas públicas y privadas, centros de educación superior que están formando a los futuros agentes sociales, y comunidades sociales y escenarios donde se construyen las políticas públicas y educativas buscando trasformaciones sociales a través de su identidad, carisma y sistema pedagógico personalizado, interdisciplinario y garante de los derechos fundamentales de las personas.

No queda más que decir que hoy existe una forma particular y pedagógica de educación, que convoca a los agentes dinamizadores educativos a un compromiso social, que se ratifica en las palabras del expresidente uruguayo:

La enseñanza del futuro está de la mano no solo de la capacidad, sino del grado de compromiso que tengan los maestros y los profesores, en trabajar sobre la cabeza y el corazón de las generaciones que vienen. El gran desafío y la gran pregunta es ¿van a estar a la altura de la enorme responsabilidad? (Mujica, 2016).

\section{Referencias}

Álvarez, C. y González, E. M. (2002). En ti, el universo. En Lecciones de didáctica general (pp. 77-91). Bogotá: Magisterio.

Asamblea General de las Naciones Unidas. (1989). Convención sobre los Derechos del Niño. Recuperado de https://www.unicef.org/argentina/spanish/7.-convencionsobrelosderechos.pdf.

Berberián, S. (12 de abril de 2015). Triste buscando culpables. Prensa Libre. Recuperado de http://www.prensalibre.com/opinion/triste-buscando-culpables.

Colombia, Congreso de la República. Constitución política de Colombia (4 de julio de 1991).

Colombia, Congreso de la República. Ley 115 (8 de febrero de 1994).

Colombia, Congreso de la República. Ley 1098 (8 de noviembre de 2006).

Colombia, Congreso de la República. Ley 1878 (9 de enero de 2018).

Durán, E., Guáqueta, C. A. y Torres, A. (2011). Restablecimiento de derechos de niños, niñas y adolescentes en el sistema nacional de bienestar 
familiar. Revista Latinoamericana de Ciencias Sociales, Niñez y Juventud, 2(9), 549-559.

Feito, L. (2007). Vulnerabilidad. Anales del Sistema Sanitario de Navarra, 30(3), 7-22. Recuperado de https://recyt.fecyt.es//index.php/AssN/article/view/2004/1431.

Flórez, R. (2005). Pedagogía y currículo. En Pedagogía del conocimiento (pp. 291-332). Bogotá: McGraw-Hill.

Moreu, A. C. (2006). La recepción de las doctrinas correccionalistas en España: políticas educativas y metodologías psicopedagógicas. Revista de Educación, 340, 755-785. Recuperado de http://www.revistaeducacion.mec.es/re340_27.html.

Mujica, J. (2010). Discurso de asunción como presidente de la República del Uruguay ante la Asamblea General del Parlamento Nacional: "Patria con todos y para todos". Relaciones Internacionales, 38, 1-22. Recuperado de http://sedici.unlp.edu.ar/bitstream/handle/10915/46681/Rep\%C3\%BAblica_Oriental_del_Uruguay_-_Discurso_de_asunci\%C3\%B3n_del_ presidente_Jos\%C3\%A9_Mujica_22_p._.pdf?sequence $=24$

Mujica, J. (7 de agosto de 2014). No le pidamos al docente arreglar los agujeros que hay en el hogar. Presidencia República Oriental de Uruguay. Recuperado de https:/wwww.presidencia.gub.uy/comunicacion/ comunicacionnoticias/utu-colonia-nicolich-mujica-inauguracion.

Mujica, J. (2016). Charla Oficina de la OEl-Mercosur con el expresidente de Uruguay, José "Pepe" Mujica, mensaje a los educadores. Uruguay. Recuperado en https://www.youtube.com/watch?v=BsYziBzRDwu.

Resolución 1519 de 2016 [Instituto Colombiano de Bienestar Familiar]. Por la cual se aprueba el lineamiento técnico del modelo para la atención de los niños, las niñas y adolescentes, con derechos inobservados, amenazados o vulnerados. 23 de febrero de 2016. Recuperado de https://www.icbf.gov.co/misionales/proteccion/restablecimiento-de-derechos

Zuluaga, D. (2017). Presentación. Perseitas, 5(1), 8-10. Recuperado de http:// funlam.edu.co/revistas/index.php/perseitas/article/view/2234/1684. 


\section{Anexo 1. Referencias incluidas en la revisión}

Abad, M. I. (2016). Proyección actual de la pedagogía amigoniana. La resiliencia como marco de acción (tesis doctoral). Universidad Complutense de Madrid, España. Recuperado de http://eprints.ucm. es/40351/1/T38083.pdf.

Amigó, L. (1986). Obras completas. Madrid: Biblioteca de Autores Cristianos de Editorial Católica.

Anno, J. (2015). Pedagogía amigoniana. Experiencia en Alemania. En III Congreso Nacional de Pedagogía Amigoniana (pp. 127-128). Madrid: Surgam.

Arive, J. (2015). Intervención pedagógica y pedagogía amigoniana. En III Congreso Nacional de Pedagogía Amigoniana (pp. 45-64). Madrid: Surgam.

Calderón, J. J. (2015). Aportaciones de la pedagogía amigoniana. Experiencia en Colombia. En III Congreso Nacional de Pedagogía Amigoniana (pp. 127-128). Madrid: Surgam.

González, A. y Vives, J. A. (1986). Introducción. En Obras completas (pp. XXXI-XLII). Madrid: Biblioteca de Autores Cristianos de Editorial Católica.

González, F. (2004). Diccionario de la pedagogía amigoniana. Madrid: Terciarios Capuchinos Provincia Luis Amigó.

González, F. (2011). Historia y desarrollo de la ficha biopsicopedagógica en los centros para jóvenes de la Congregación de Religiosos Terciarios Capuchinos. Psychologia Latina, 2(1), 47-108.

Hoffend. J. (2015). Pedagogía amigoniana. Experiencia en Polonia. En III Congreso Nacional de Pedagogía Amigoniana. Madrid: Surgam.

León, O. U., Calderón, J. J. y Romero, S. P. (2010). Aproximación a los referentes teóricos y conceptuales que fundamentan la propuesta pedagógica amigoniana. Oficina Provincial de Comunicaciones, Bogotá.

Loizaga, F. (2015). Apego y vínculo seguros: ¿qué pilares sustentan la educación y la parentalidad positiva? En III Congreso Nacional de Pedagogía Amigoniana (pp. 119-121). Madrid: Surgam Editorial .

Lostado, J. A. (2015). Discurso inaugural. En III Congreso Nacional de Pedagogía Amigoniana (pp. 11-20). Madrid: Surgam.

Marina, J. A. (2015). El educador, comprendiendo el presente y mirando el futuro. En III Congreso Nacional de Pedagogía Amigoniana (pp. 123124). Madrid: Surgam.

Martínez, C. (2015). Discurso inaugural. En II Congreso Nacional de Pedagogía Amigoniana (pp. 21-23). Madrid: Surgam.

Martínez, M. (2008). Los principios amigonianos en la práctica pedagógica. Revista Surgam, 59(501), 1-24. Recuperado de http://www. surgam.org/articulos/501/. 
Martínez, M. (2009). Lección inaugural una práctica hecha pedagogía nació del espíritu para hacerse academia. En III Congreso Internacional de Pedagogía Amigoniana (pp. 1-31). Recuperado de http://files.cursosbiblianixon.webnode.com.co/200000178-2d8882f7d2/12_01_ LECCION.INAUGURAL.LA.PEDAGOGIA.AMIGONIANA.pdf.

Martínez, M. (2015). Un carisma evangélico que se actualiza y atiende desafíos. En III Congreso Nacional de Pedagogía Amigoniana (pp. 3132). Madrid: Surgam.

Mestre, M. V. (2015). La psicología aplicada en la pedagogía amigoniana: raíces y actualidad en el contexto social. En III Congreso Nacional de Pedagogía Amigoniana (pp. 39-44). Madrid: Surgam.

Mestre, M. V., Sánchez, J., Ridaura, J., Bello, J. M., López, P. y Navarro Pérez, J. J. (2012). Aportación de los Terciarios Capuchinos a la Psicología y Psicometría española. Revista Historia de la Psicología, 33, 57-74.

Montero, A. (2015). Influencia de la pedagogía amigoniana en el derecho de la infancia. En III Congreso Nacional de Pedagogía Amigoniana (pp. 65-82). Madrid: Surgam.

Ortiz, L. y Fagua, W. (2015). Friendship home father Luis Amigó. En III Congreso Nacional de Pedagogía Amigoniana (pp. 177-181). Madrid: Surgam.

Osoro, C. (2015). Discurso inaugural. En III Congreso Nacional de Pedagogía Amigoniana (pp. 33-35). Madrid: Surgam.

Peral del, R. (2015). Aportaciones de la pedagogía amigoniana en España. En III Congreso Nacional de Pedagogía Amigoniana (p. 127). Madrid: Surgam.

Pérez, F. G. (2015). Aportaciones de la pedagogía amigoniana. Experiencia en México. En III Congreso Nacional de Pedagogía Amigoniana. Recuperado de https://www.youtube.com/watch?v=pa9XGqk5LzY\&t=34s.

Religiosos Terciarios Capuchinos. (2012). Ruta de la pedagogía Amigoniana. Bogotá: Centro de Investigaciones Provincial San José "Carisma y Saber".

Roa, G. Y. (2014). El estudio de casos. Religiosos Terciarios Capuchinos de Nuestra Señora de los Dolores. Provincia San José.

Rodríguez, M. I. (2015). Aportaciones de la pedagogía. Experiencia en Argentina. En III Congreso Nacional de Pedagogía Amigoniana. Recuperado de https://www.youtube.com/watch?v=pa9XGqk5LzY\&$\mathrm{t}=34 \mathrm{~s}$.

Sánchez, V., Guijarro, T. y Sanz, Y. (2005). La observación psicológica en los tribunales para niños en España (1889-1975). (I) los Terciarios Capuchinos y la psicología. Revista de la Asociación Española de Neuropsiquiatría, 94, 113-129. Recuperado de http://scielo.isciii.es/ scielo.php?script=sci_arttext\&pid=S0211-57352005000200007. 
Sesé, A. (2015). La originalidad de la pedagogía amigoniana. En III Congreso Nacional de Pedagogía Amigoniana (pp. 97-100). Madrid: Surgam.

Terciarios Capuchinos. (1985). Manual pedagógico de los Terciarios Capuchinos. Valencia: Surgam.

Tobón, F. (2015). Fundación Amigó: misión compartida, trabajo en red e innovación socioeducativa. En III Congreso Nacional de Pedagogía Amigoniana. Recuperado de https://www.youtube.com/watch?v=MUR-hj2uUgw\&t=69s.

Torres, J., y Vélez, I. (2015). Aportaciones de la pedagogía amigoniana. Experiencia en Puerto Rico. En III Congreso Nacional de Pedagogía Amigoniana. Recuperado de https:/www.youtube.com/watch?$\mathrm{v}=$ pa9XGqk5LzY\&t=34s.

Velasco, A. P. (2016). Ruta de la pedagogía amigoniana: experiencia investigativa basada en la cartografía social. Boletín Redipe, 5(9), 1-12. Recuperado de http://revista.redipe.org/index.php/1/article/ view/112.

Vélez, C. (2009). Pasado, presente y futuro de la pedagogía reeducativa. En III Congreso Internacional de Pedagogía Amigoniana (pp. 1-23). Recuperado de http://www.funlam.edu.co/uploads/eventosfunlam/12_24_LA.PEDAGOGIA.REEDUCATIVA.PASADO\%96PRESENTE\%96PROSPECTIva.pdf.

Vélez, C., Ariza, N. P., Calderón, J. J., Ramírez, L. E., Hernández, M. A. y González, A, (2008). Saberes, actores y escenarios de la Licenciatura en Pedagogía Reeducativa (1984 a 2008). Bogotá: Fundación Universitaria Luis Amigó.

Venceslao, M. (2012). Pedagogía correccional estudio antropológico sobre un Centro Educativo de Justicia Juvenil (tesis doctoral). Universitat de Barcelona, España. Recuperado de: http://diposit.ub.edu/ dspace/bitstream/2445/35239/5/MVP_TESIs.pdf.

Vives, J. A. (2000). Identidad amigoniana en acción. Medellín: Funlam.

Vives, J. A. (2001). Identidad amigoniana en acción (2. ${ }^{\mathrm{a}}$ ed.). Valencia: Funlam.

Vives, J. A. (2015). Carisma y pedagogía. En III Congreso Nacional de Pedagogía Amigoniana (pp. 83-95). Madrid: Surgam.

Zambrano, A. (2015). Pedagogía y didáctica: esbozo de las diferencias, tensiones y relaciones de dos campos. Praxis y Saber, 7(13), 45-61. Recuperado de http://www.scielo.org.co/pdf/prasa/v7n13/ v7n13a03.pdf. 